\title{
Über schwefligmolybdänsaure Salze.
}

\author{
Von \\ Arthur Rosenheim. \\ II. Mitteilung. 1
}

Bei der Untersuchung der von Péchard ${ }^{2}$ zuerst dargestellten schwefligmolybdänsauren Salze waren, wie in der I. Mitteilung ${ }^{3}$ veröfentlicht wurde, Resultate erhalten worden, die von denen des Entdeckers wesentlich abwichen. Während dieser für die durch Einwirkung gasförmiger schwefliger Säure auf die Lösungen molybdänsaurer Salze erhaltenen Verbindungen die Zusammensetzung

$$
4 \mathrm{R}_{2} \mathrm{O}_{3} 3 \mathrm{SO}_{2} \cdot 10 \mathrm{MO}_{3}+\mathrm{x} \text { aq }\left(\mathrm{R}=\mathrm{K}, \mathrm{Na}, \mathrm{NH}_{4}\right)
$$

erhielt, ergab die Wiederholung der Versuche für die drei untersuchten Alkalisalze abweichende Formeln, nämlich

$$
\begin{aligned}
& 3\left(\mathrm{NH}_{4}\right)_{2} \mathrm{O} .2 \mathrm{SO}_{2} \cdot 8 \mathrm{MO}_{3}+5 \mathrm{H}_{2} \mathrm{O} . \\
& 4 \mathrm{~K}_{2} \mathrm{O} .4 \mathrm{SO}_{2} \cdot 9 \mathrm{MO}_{3}+5 \mathrm{H}_{2} \mathrm{O} . \\
& 9 \mathrm{Na}_{2} \mathrm{O} .8 \mathrm{SO}_{2} \cdot 20 \mathrm{MO}_{3}+37 \mathrm{H}_{2} \mathrm{O} .
\end{aligned}
$$

Dies merkwürdige Resultat bei den auf ganz analogem Wege durch Einwirkung von schwefliger Säure auf die Lösungen der Alkaliparamolybdate erhaltenen Salzen, wurde damals auf den Einflufs der Basis, die unzweifelhaft oft von grofser Bedeutung für die Zusammensetzung des in komplexen Salzen enthaltenen sauren Ions ist, zurückgeführt.

1 Die Ausfïhrung der hiermit publizierten Versuche war im Sommersemester 1895 Herrn stud. D. Asch übertragen worden, der die Arbeit zu Promotionszwecken verwenden wollte. Aus äufseren Gründen war ich gezwungen grenanntem Herrn das Thema zu entziehen, trotzdem er schon längere Zeit daran gearbeitet hatte, und veröffentliche ich nunmehr nach vollständiger Neubearbeitung die erhaltenen Ergebnisse, da ich dieselben im Zusammenlang mit anderen von mir ausgeführten und demnächst zu veröffentlichenden Versuchen verwerten muls.

2 Compt. rend. 116, 1441-1444. Ann. Chim. Phys. [6] 30, 395.

3 Diese Zeitschr. 7, 176-184. 
Es war deshalb von Wichtigkeit uuch andere Verbindungen der Schwefligmolybdänsäure zu untersuchen und ferner zu prüfen, ob man durch Einwirkung von schwefliger Säure auf andere Molybdate als die Paraverbindungen, wieder anders zusammengesetzte komplexe Salze erhalten würde.

Seit der Veröffentlichung der I. Mitteilung war die Analyse der vorliegenden Verbindungen durch die ausgezeichnete jodometrische Molybdänsäurebestimmung von FrIEDHExM und EULER ${ }^{1}$ wesentlich erleichtert worden, so dafs auch eine Kontrolle der früher erhaltenen Ergebnisse angebracht erschien. Hierbei zeigte es sich, dafs die gewichtsanalytische Bestimmung der Molybdänsäure in den schweflignolybdänsauren Salzen der fixen Alkalien mit einem konstanten Fehler behaftet war, der das Bild der Formeln wesentlich verschoben hatte, und dafs sich nunmehr die Resultate viel einfacher stellten. Ein Teil der Molybdänsäure scheint aufserordentlich fest mit der schwefligen Säure verbunden zu sein und sich dadurch der Fällung durch Schwefelwasserstoff zu entziehen. Trotzdem bei den früheren Analysen die schweflige Säure durch anhaltendes Kochen mit Schwefelsäure scheinbar vertrieben war, bevor man Schwefelwasserstoff zur Fällung des. Molybdänsulfides in die Lösung einleitete, wurden doch bei allen derartig ausgeführten Analysen - und ibre Zahl war der merkwürdigen Zusammensetzung der Salze halber eine sehr grofse - wie sich jetzt zeigte, stets zu niedrige Werte erhalten.

\section{Einwirkung von schwefliger Säure auf paramolybdänsaure Salze.}

Von den Salzen der fixen Alkalien wurden untersucht die Natrium-, Kalium-, Lithium-, Rubidium- und Cäsiumverbindung. Sämtliche Paramolybdate wurden dargestellt, indem 1 Mol. des Alkalikarbonates in Wasser gelöst mit ungefähr 2 Mol. Molybdänsäure gekocht wurden, bis die letztere sich aufgelöst hatte. Aus der eingeengten Lösung schieden sich die Paramolybdate in reichlicher Ausbeute ab. Nur das Kaliumsalz zersetzte sich hierbei in 'Trimolybdat und wurde deshalb besser durch Einwirkung von verdünnter Salpetersäure auf eine Lösung des normalen Molybdates nach den in der I. Mitteilung gemachten Angaben gewonnen. Das Cäsium- und Rubidiumparamolybdat, beides recht schwerlösliche Salze, schieden sich schon aus verdünnten Lösungen krystallinisch

1 Ber, deutsch. chem. Ges. 28, 2061-2067. 
ab. Das Lithiumparamolybdat dagegen krystallisierte erst aus syrupöser Lösung über Phosphorpentoxyd in weilsen Nädelchen aus. Die Analyse dieses bisher noch nicht beschriebenen Salzes ergab für dasselbe die Zusammensetzung

$3 \mathrm{Li}_{2} 0.7 \mathrm{MO}_{3}+12 \mathrm{H}_{2} \mathrm{O} .{ }^{.1}$

\begin{tabular}{|c|c|c|c|c|}
\hline $\begin{array}{c}\text { Angewandte } \\
\text { Substanz } \\
\mathrm{g}\end{array}$ & $\begin{array}{c}\text { Gefunden } \\
\mathrm{g} \\
\end{array}$ & Prozente & Mittel & Berechnet \\
\hline $\begin{array}{l}0.5899 \\
0.4740 \\
0.2038 \\
0.2078\end{array}$ & $\begin{array}{l}0.1426 \mathrm{Ji}_{2} \mathrm{SO}_{4} \\
0.1398 \mathrm{Li}_{2} \mathrm{SO}_{4} \\
0.1577 \mathrm{MO}_{3} \\
0.1603 \mathrm{MO}_{3}\end{array}$ & $\begin{array}{r}6.59 \mathrm{Li}_{2} \mathrm{O} \\
6.64 \mathrm{Li}_{2} \mathrm{O} \\
77.38 \mathrm{MO}_{2} \\
77.14 \mathrm{MO}_{3}\end{array}$ & $\begin{array}{l}\left\{6.62 \% \mathrm{Li}_{2} \mathrm{O}\right. \\
77.21 \% \mathrm{MO}_{3}\end{array}$ & $\begin{array}{r}6.85 \% \mathrm{Li}_{2} \mathrm{O} \\
76.71 \% \mathrm{MO}_{3}\end{array}$ \\
\hline & & Differenz & $16.17 \% \mathrm{H}_{2} \mathrm{O}$ & $16.44 \% \mathrm{H}_{22} \mathrm{O}$ \\
\hline
\end{tabular}

Zur Darstellung der entsprechenden schwefligmolybdänsauren Salze wurden die möglichst gesättigten Lösungen der Paramolybdate bei gewöhnlicher Temperatur mit gasförmiger schwefliger Säure gesättigt. Das Kali- und Natronsalz wurden in den in der I. Mitteilung schon beschriebenen Formen erhalten. Das Cäsium- sowie das Rubidiumsalz entsprachen im Verhalten und Aussehen ganz dem Kalisalz. Ein Lithiumsalz konnte in reinem Zustande nicht gewonnen werden, da es leichter löslich war als das gleichzeitig auskrystallisierende Sulfit, und sich die Lösung bei der dazu notwendigen Konzentration stark zersetzte.

Die Analysen sämtlicher Salze ${ }^{2}$ führten zu der allgemeinen Formel

$$
2 \mathrm{R}_{2} 0.2 \mathrm{SO}_{2} .5 \mathrm{MO}_{3}+\mathrm{xaq}
$$

die sich von der Pfehard'schen um 1/2 Mol. schwefliger Säure unterscheidet, was jedoch analytisch sehr stark zum Ausdruck kommt.

Die einzelnen Ergebnisse sind die folgenden.

1 Analyse ausgeführt von D. Asch.

${ }^{2}$ Molybdänsäure wurde, nach Wegkochen der schwefligen Säure in salzsaurer Lösung, jodometrisch nach FriedHerm und Euler (l. c.), bestimmt. Zur Alkalibestimmung wurden die Säuren dureh Quecksilberoxydulnitrat und Quecksilberoxyd entfernt und aus dem Filtrat, nach Ausfällung der überschüssigen Quecksilbersalze, das Alkali als Sulfat gewonnen. Schweflige Säure wurde durch Brom und Alkali oxydiert und als Baryumsulfat zur Wägung gebracht. 
I. $2 \mathrm{Na}_{2} 0.2 \mathrm{SO}_{2} .5 \mathrm{MO}_{3}+8 \mathrm{H}_{2} \mathrm{O}$.

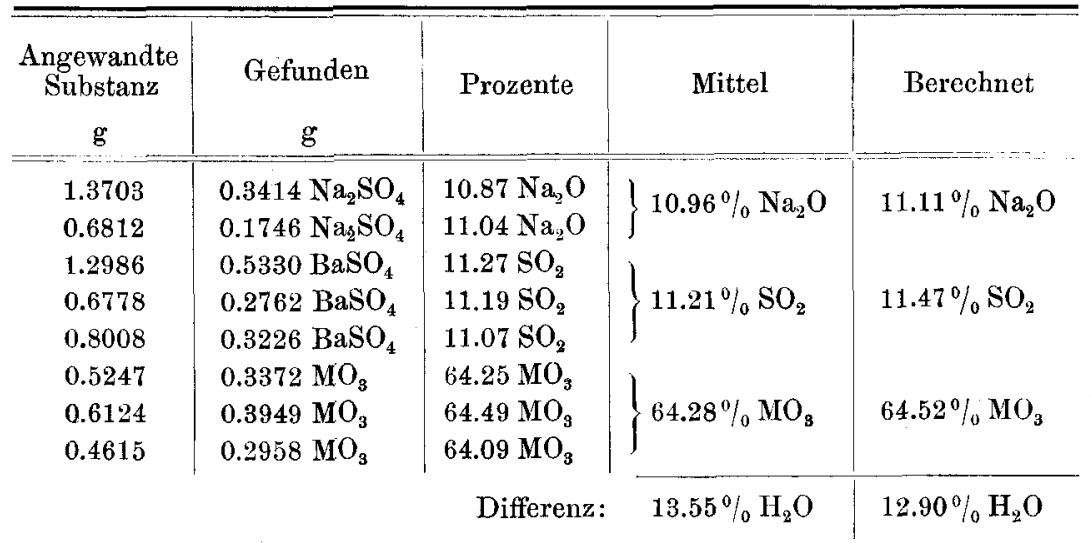

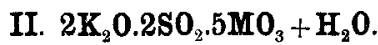

\begin{tabular}{c|c|c|c|c}
\hline $\begin{array}{c}\text { Angewandte } \\
\text { Substanz }\end{array}$ & Gefunden & Prozente & Mittel & Berechnet \\
$\mathrm{g}$ & $g$ & & & \\
\hline & & & & \\
0.2516 & $0.0862 \mathrm{~K}_{2} \mathrm{SO}_{4}$ & $18.53 \mathrm{~K}_{2} \mathrm{O}$ & $\left\{18.36 \% \mathrm{~K}_{2} \mathrm{O}\right.$ & $18.53 \% \mathrm{~K}_{2} \mathrm{O}$ \\
0.3951 & $0.1330 \mathrm{~K}_{2} \mathrm{SO}_{4}$ & $18.20 \mathrm{~K}_{2} \mathrm{O}$ & \} & \\
0.6971 & $0.3064 \mathrm{BaSO}_{4}$ & $12.07 \mathrm{SO}_{2}$ & \} $11.98 \% \mathrm{SO}_{2}$ & $12.04 \% \mathrm{~K}_{2} \mathrm{O}$ \\
0.4653 & $0.2014 \mathrm{BaSO}_{4}$ & $11.89 \mathrm{SO}_{2}$ & \} & \\
0.3352 & $0.2276 \mathrm{MO}_{3}$ & $67.93 \mathrm{MO}_{3}$ & $68.01 \% \mathrm{MO}_{3}$ & $67.64 \% \mathrm{MO}_{3}$ \\
0.3513 & $0.2392 \mathrm{MO}_{3}$ & $68.08 \mathrm{MO}_{3}$ & & \\
& & Differenz: & $1.65 \% \mathrm{H}_{2} \mathrm{O}$ & $1.69 \% \mathrm{H}_{2} \mathrm{O}$
\end{tabular}

III. $2 \mathrm{Rb}_{2} 0.2 \mathrm{SO}_{2} .5 \mathrm{MO}_{3}+{ }^{1 / 2} \mathrm{H}_{2} \mathrm{O}$.

\begin{tabular}{|c|c|c|c|c|}
\hline $\begin{array}{l}\text { Angewandte } \\
\text { Substanz }\end{array}$ & & Prozente & Mittel & Berechnet \\
\hline$g$ & $g$ & & & \\
\hline 0.5289 & $0.2300 \mathrm{Rb}_{2} \mathrm{SO}_{4}$ & $30.46 \mathrm{Rb}_{2} \mathrm{O}$ & \multirow{2}{*}{$30.55 \% \mathrm{Rb}_{2} \mathrm{O}$} & \multirow{2}{*}{$30.27 \% \mathrm{Rb}_{2} \mathrm{O}$} \\
\hline 0.4814 & $0.2106 \mathrm{Rb}_{2} \mathrm{SO}_{4}$ & $30.64 \mathrm{Rb}_{2} \mathrm{O}$ & & \\
\hline 0.5999 & $0.2257 \mathrm{BaSO}_{4}$ & $10.33 \mathrm{SO}_{2}$ & \multirow{2}{*}{$10.39 \% \mathrm{SO}_{2}$} & \multirow{2}{*}{$10.41 \% \mathrm{SO}_{2}$} \\
\hline 0.4583 & $0.1743 \mathrm{BaSO}_{4}$ & $10.46 \mathrm{SO}_{2}$ & & \\
\hline 0.5286 & $0.3087 \mathrm{MO}_{3}$ & $58.40 \mathrm{MO}_{3}$ & \multirow{2}{*}{$58.34 \% \mathrm{MO}_{3}$} & \multirow{2}{*}{$58.58 \% \mathrm{MO}_{3}$} \\
\hline 0.3233 & $0.1884 \mathrm{MO}_{3}$ & $58.28 \mathrm{MO}_{3}$ & & \\
\hline \multicolumn{3}{|r|}{ Differenz: } & $0.72 \% \mathrm{H}_{2} \mathrm{O}$ & $0.74 \% \mathrm{H}_{2} \mathrm{O}$ \\
\hline
\end{tabular}


IV. $2 \mathrm{Cs}_{2} 0.2 \mathrm{SO}_{2} .5 \mathrm{MO}_{3}+6 \mathrm{H}_{2} \mathrm{O}$.

\begin{tabular}{|c|c|c|c|c|}
\hline $\begin{array}{c}\text { Angewandte } \\
\text { Substanz } \\
g\end{array}$ & $\begin{array}{l}\text { Gefunden } \\
\mathrm{g}\end{array}$ & Prozente & Mittel & Berechnet \\
\hline $\begin{array}{l}0.5765 \\
0.7695 \\
0.5525 \\
0.5491\end{array}$ & $\begin{array}{l}0.2730 \mathrm{Cs}_{2} \mathrm{SO}_{4} \\
0.2321 \mathrm{BaSO}_{4} \\
0.2629 \mathrm{MO}_{3} \\
0.2607 \mathrm{MO}_{3}\end{array}$ & $\begin{array}{c}36.89 \mathrm{Cs}_{2} \mathrm{O} \\
8.28 \quad \mathrm{SO}_{2} \\
47.59 \quad \mathrm{MO}_{3} \\
47.47 \quad \mathrm{MO}_{3}\end{array}$ & $\begin{array}{c}36.89 \% \mathrm{Cs}_{2} \mathrm{O} \\
8.28 \% \mathrm{SO}_{2} \\
47.53 \% \mathrm{MO}_{3}\end{array}$ & $\begin{array}{c}37.11 \% \mathrm{Cs}_{2} \mathrm{O} \\
8.42 \% \mathrm{SO}_{2} \\
47.37 \% \mathrm{MO}_{3}\end{array}$ \\
\hline & & Differenz & $7.30 \% \mathrm{H}_{2} \mathrm{O}$ & $7.10 \% \mathrm{H}_{2} \mathrm{O}$ \\
\hline
\end{tabular}

Sïmtliche erhaltenen Salze zersetzten sich in der Mutterlauge belassen nach einiger Zeit unter Abscheidung des entsprechenden Trimolybdates. In trockenem Zustande aufbewahrt gaben sie allmählich schweflige Säure $a b$ und verwitterten alsbald.

Durch Sättigung einer verdünnten Lösung von Ammoniumparamolybdat mit schwefliger Säure und langsame Krystallisation über Schwefelsäure wurde das in der I. Mitteilung beschriebene Salz $3\left(\mathrm{NH}_{4}\right)_{2} 0.2 \mathrm{SO}_{2} .8 \mathrm{MO}_{3}+5 \mathrm{H}_{2} \mathrm{O}$ erhalten, dessen Formel durch erneute Analysen bestätigt wurde. ${ }^{1}$

Sättigte man jedoch eine konzentrierte Lösung des Paramolybdates mit schwefliger Säure unter Kühlung, so konnte man bemerken, dals anfangs, sowie die Lösung nahezu gesättigt war, sich schneeweise, lange Nadeln abschieden, die teilweise mit den oktaedrischen gelblichen Krystallen des obigen Salzes durchsetzt waren und bei weiterem Einleiten der schwefligen Säure alsbald in Lösung gingen. Die ahgesaugten Nadeln, die in trockenem Zustande aufserordentlich stark schweflige Säure abgaben, konnten auf diesem Wege nicht analysenrein erhalten werden. Wiederholte Untersuchungen ergaben sehr wechselnde Werte.

Es wurde deshalb eine sehr konzentrierte mit schwefliger Säure stark übersättigte Lösung von Natriumparamolybdat mit einer kaltgesättigten Lösung von Chlorammonium versetzt. Sofort erstarrte die ganze Masse zu einem Gemisch der weifsen langen Nadeln. Dieselben wurden schnell abgesaugt mit kaltem Wasser, Alkohol and Ather gewaschen und sofort zur Analyse verwendet. Diese ergab für das Salz eine den vorigen Verbindungen entsprechende Zusammensetzung.

1 Die Molybdänsäurebestimmung war durch einfaches Abrösten gewogener Mengen des Salzes bewirkt worden, so dafs der Analysenfehler, der die Formel der anderen Salze beeinflulst hatte, hier nicht einwirken konnte. 
$2\left(\mathrm{NH}_{4}\right)_{2} 0.2 \mathrm{SO}_{2} .5 \mathrm{MO}_{8}+12 \mathrm{H}_{2} \mathrm{O}$.

\begin{tabular}{|c|c|c|c|c|}
\hline $\begin{array}{c}\text { Angewandte } \\
\text { Substanz } \\
g \\
\end{array}$ & $\begin{array}{c}\text { Gefunden } \\
\mathrm{g}\end{array}$ & Prozente & Mittel & Berechnet \\
\hline $\begin{array}{l}0.8210 \\
1.0476 \\
0.6020 \\
1.1249 \\
0.8484 \\
1.1907\end{array}$ & $\begin{array}{l}0.2750 \mathrm{Pt} \\
0.3466 \mathrm{Pt} \\
0.2402 \mathrm{BaSO}_{4} \\
0.4491 \mathrm{BaSO}_{4} \\
0.5269 \mathrm{MO}_{3} \\
0.7323 \mathrm{MO}_{3}\end{array}$ & $\begin{array}{c}8.93\left(\mathrm{NH}_{4}\right)_{2} \mathrm{O} \\
8.82\left(\mathrm{NH}_{4}\right)_{2} \mathrm{O} \\
10.96 \mathrm{SO}_{2} \\
10.97 \mathrm{SO}_{8} \\
62.10 \mathrm{MO}_{3} \\
61.50 \mathrm{MO}_{3}\end{array}$ & $\begin{array}{l}8.87 \%\left(\mathrm{NH}_{4}\right)_{2} \mathrm{O} \\
10.97 \% \mathrm{SO}_{2} \\
61.80 \% \mathrm{MO}_{3}\end{array}$ & $\begin{array}{l}10.96 \% \mathrm{SO}_{2} \\
61.84 \% \mathrm{MO}_{3}\end{array}$ \\
\hline & & Differenz: & $18.36 \% \mathrm{H}_{2} \mathrm{O}$ & $18.50 \% \mathrm{H}_{2} \mathrm{O}$ \\
\hline
\end{tabular}

Weitere Versuche, durch Einwirkung von schwefliger Säure auf die Paramolybdate der Erdalkalien die entsprechenden schwefligmolybdänsauren Salze zu erhalten, verliefen resultatlos. Die an und für sich in Wasser unlöslichen Paramolybdate lösten sich zwar in Wasser suspendiert beim Einleiten des Schwefligsäuregases auf, doch ergab die Krystallisation der Lösung nur Mischungen von nicht konstanter Zusammensetzung. Durch Umsetzung einer mit schwefliger Säure abgesättigten Lösung des Natriumparamolybdates mit kaltgesättigten Lösungen der Erdalkalichloride wurden jedoch bei langsamer Krystallisation über Schwefelsäure schöne prismatische Krystalle eines Baryum- und Strontiumsalzes, sowie ein in Nadeln krystallisierendes Calciumsalz dargestellt. Das letztere wurde seiner sehr grofsen Löslichkeit halber nie ganz analysenrein erhalten, sondern schlofs stets bedeutende Mengen der syrupösen Mutterlauge ein. Die Analysen des Baryum- und Strotiumsalzes führten zu den folgenden Ergebnissen:

$2 \mathrm{BaO}^{2} .2 \mathrm{SO}_{2} .5 \mathrm{MO}_{3}+10 \mathrm{H}_{2} \mathrm{O}$.

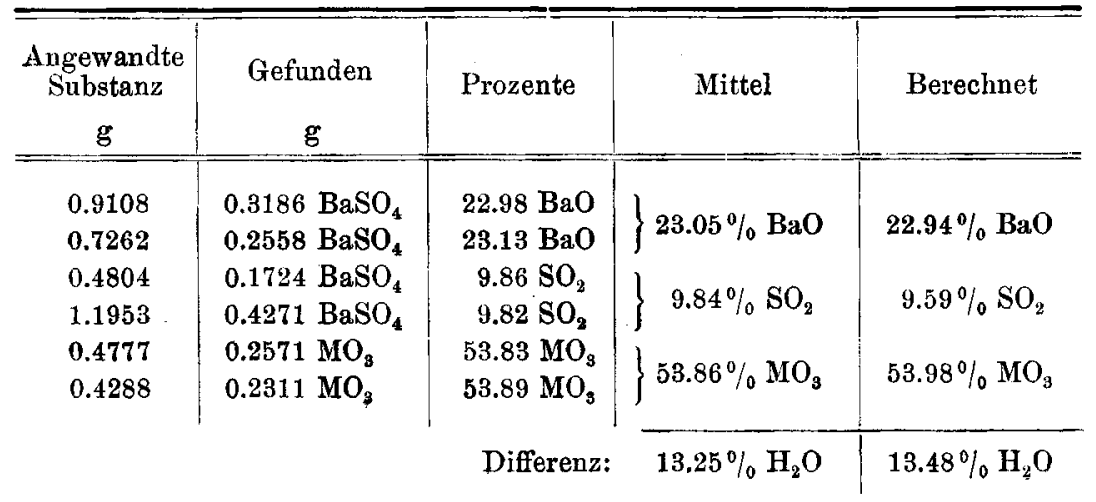


$2 \mathrm{Sr} 0.2 \mathrm{SO}_{2} .5 \mathrm{MO}_{3}+12 \mathrm{H}_{2} \mathrm{O}$.

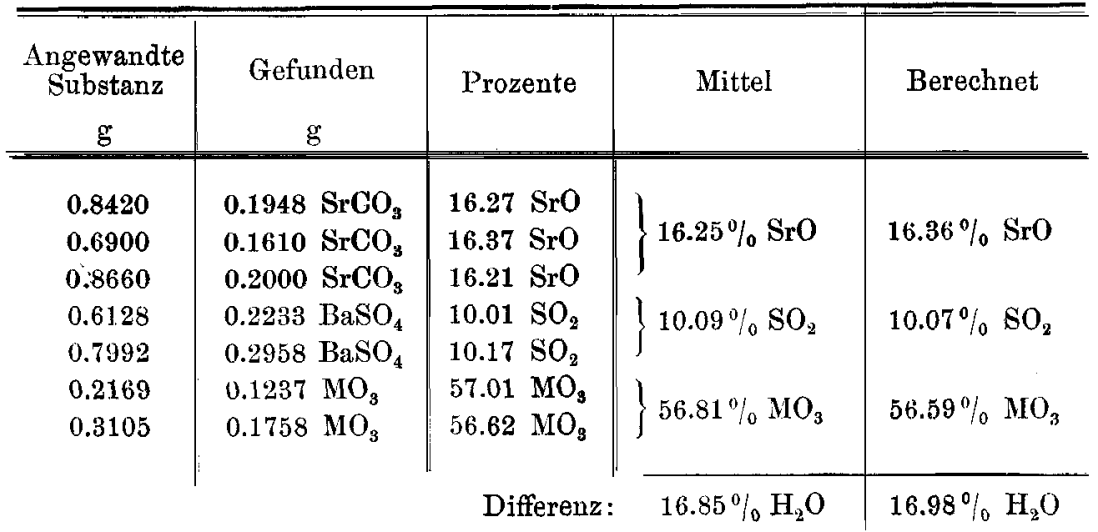

Aus dem auch in Lösung beständigen Ammoniumsalze $3\left(\mathrm{NH}_{4}\right)_{2} \mathrm{O}$. $2 \mathrm{SO}_{2} .8 \mathrm{MO}_{3}+5 \mathrm{H}_{2} \mathrm{O}$ analoge Salze anderer Basen durch doppelte Umsetzung zu erhalten, gelang bisher nicht. Bei den meisten Versuchen krystallisierte das Ammoniumsalz unverändert wieder aus, und in anderen Fällen, z. B. bei der Umsetzung mit Kaliumchlorid, trat unter Abscheidung des betreffenden Trimolybdates Zersetzung ein.

\section{Einwirkung von schwefliger Säure auf normale molybdänsaure Salze.}

Wurden Lösungen der normalen molybdänsäuren Salze von der Zusammensetzung $\mathrm{R}_{2}^{1} \mathrm{O} . \mathrm{MO}_{3}$ mit schwefliger Säure vollständig abgesättigt, so krystallisierten in allen Fällen dieselben Körper aus, wie bei Einwirkung anf die analogen Paramolybdate. Wurde dagegen nicht bis zur vollständigen Absättigung das Gas eingeleitet, so krystallisierten neben den schwefligmolybdänsauren Salzen die entsprechenden schwefligsäurefreien Paramolybdate aus. Hieraus ergiebt sich, dals durch die Anwesenheit einer grölseren Basismenge der Reaktionsverlauf nicht geändert wird. Aus normalem Molybdat bildet sich primär Paramolybdat, das sekundär durch Aufnahme von schwefliger Säure in ein komplexes Molybdänsulfit übergeht. 


\section{Einwirkung von schwefliger Säure auf tetramolybdänsaure Salze.}

Dagegen verlief die Reaktion wesentlich anders, sowie Molybdate angewendet wurden, die weniger Basis enthielten, als die Salze der Parareihe. Wurden Lösungen von tetramolybdänsauren Salzen $\mathrm{R}_{2}^{1} \mathrm{O}_{4} \mathrm{MO}_{3}$ mit schwefliger Säure behandelt, so entstanden keine komplexen Molybdänsulfite, sondern nur sauere Salze der Molybdänsäure, oktamolybdänsaure Salze oder, wie sie von manchen Chemikern bezeichnet werden, primäre Tetramolybdate.

Die Lösungen der Tetramolybdate des Kaliums und Natriums wurden durch Digestion gewogener Mengen der Alkalikarbonate mit berechneten Mengen Molybdänsäure in wässeriger Lösung dargestellt. Zur Darstellung des Kalisalzes darf hierbei nicht zu stark erwärmt werden, da sonst unter Zersetzung die Lösung plötzlich zu einem Gemisch weifser Nadeln von Kaliumtrimolybdat erstarrt. Das Ammoniumtetramolybdat wurde durch mehrstündiges Erhitzen fein gepulverten Ammoniumparamolybdates im Luftbad auf $150^{\circ} \mathrm{er}-$ halten. ${ }^{1}$ Es ist ein weilses, in Wasser unlösliches Pulver; seine Zusammensetzung wurde durch die Analyse bestätigt.

Beim Einleiten von schwefliger Säure in die Lösungen des Kalium- und Natriummolybdates, sowie in die Suspension des Ammoniumsalzes in Wasser, trat eine grünlichblaue Färbung infolge teilweiser Reduktion der Molybdänsäure ein. Aus den Laugen krystallisierten über Schwefelsäure die schwefligsäurefreien, etwas grünlich gefärbten Salze aus, das Kalium- und Natriumsalz in wohl ausgebildeten Prismen, das Ammoniumsalz in glänzenden prismatischen Nadeln.

Es ist dies eine bequeme Methode, um diese sonst schwer zugänglichen Salze in beliebiger Menge darzustellen. Das Kaliumund Natriumsalz ist schon von ULLIK ${ }^{2}$ erhalten worden, das Ammoniumsalz ist bisher nicht beschrieben.

Die Analysen hatten folgende Ergebnisse:

1 „Beiträge zur Kenntnis der molybdänsauren Salze“ (Inang-Dissertation, F. Westrhal, Berlin 1895, S. 15).

2 Lieb. Ann. 144, 324; 153, 372. 
$\mathrm{K}_{2} 0.8 \mathrm{MO}_{3}+13 \mathrm{H}_{2} 0 .{ }^{1}$

\begin{tabular}{|c|c|c|c|c|}
\hline $\begin{array}{c}\text { Angewandte } \\
\text { Substanz } \\
\mathrm{g}^{\circ}\end{array}$ & $\begin{array}{c}\text { Gefunden } \\
\qquad g\end{array}$ & Prozente & Mittel & Berechnet \\
\hline $\begin{array}{l}0.4354 \\
0.5437 \\
0.2022 \\
0.2734\end{array}$ & $\begin{array}{l}0.0519 \mathrm{~K}_{8} \mathrm{SO}_{4} \\
0.0636 \mathrm{~K}_{2} \mathrm{SO}_{4} \\
0.1576 \mathrm{MO}_{3} \\
0.2125 \mathrm{MO}_{3}\end{array}$ & $\begin{array}{r}6.44 \mathrm{~K}_{8} \mathrm{O} \\
6.32 \mathrm{~K}_{2} \mathrm{O} \\
77.94 \mathrm{MO}_{3} \\
77.72 \mathrm{MO}_{3}\end{array}$ & $\left\{\begin{array}{l}6.38 \% \mathrm{~K}_{2} \mathrm{O} \\
77.83 \% \mathrm{MO}_{3}\end{array}\right.$ & $\begin{array}{r}6.20 \% \mathrm{~K}_{2} \mathrm{O} \\
77.83 \% \mathrm{MO}_{3}\end{array}$ \\
\hline & & Differenz: & $15.79 \% \mathrm{H}_{2} \mathrm{O}$ & $15.97 \% \mathrm{H}_{2} \mathrm{O}$ \\
\hline
\end{tabular}

$\mathrm{Na}_{2} \mathrm{O} .8 \mathrm{MO}_{8}+15 \mathrm{H}_{2} \mathrm{O}$.

\begin{tabular}{|c|c|c|c|c|}
\hline $\begin{array}{c}\text { Angewandte } \\
\text { Substanz } \\
\mathrm{g}\end{array}$ & $\begin{array}{c}\text { Gefunden } \\
\mathrm{g}\end{array}$ & Prozente & Mittel & Berechnet \\
\hline $\begin{array}{l}0.6244 \\
0.8441 \\
0.2926 \\
0.3016\end{array}$ & $\begin{array}{l}0.0649 \mathrm{Na}_{2} \mathrm{SO}_{4} \\
0.0848 \mathrm{Na}_{2} \mathrm{SO}_{4} \\
0.2275 \mathrm{MO}_{3} \\
0.2331 \mathrm{MO}_{3}\end{array}$ & $\begin{array}{r}4.53 \mathrm{Na}_{2} \mathrm{O} \\
4.39 \mathrm{Na}_{2} \mathrm{O} \\
77.76 \mathrm{MO}_{3} \\
77.29 \mathrm{MO}_{3}\end{array}$ & $\begin{array}{l}4.46 \% \mathrm{Na}_{2} \mathrm{O} \\
77.52 \% \mathrm{MO}_{3}\end{array}$ & $\begin{array}{r}4.32 \% \mathrm{Na}_{2} \mathrm{O} \\
77.63 \% \mathrm{MO}_{3}\end{array}$ \\
\hline & & Differenz: & $18.02 \% \mathrm{H}_{2} \mathrm{O}$ & $18.05 \% \mathrm{H}_{2} \mathrm{O}$ \\
\hline
\end{tabular}

$\left(\mathrm{NH}_{4}\right)_{2} 0.8 \mathrm{MO}_{3}+13 \mathrm{H}_{2} \mathrm{O}$.

\begin{tabular}{|c|c|c|c|c|}
\hline $\begin{array}{c}\text { Angewandte } \\
\text { Substanz } \\
\mathrm{g}\end{array}$ & $\begin{array}{c}\text { Gefunden } \\
\mathrm{g}\end{array}$ & Prozente & Mittel & Berechnet \\
\hline $\begin{array}{l}0.4422 \\
0.2000 \\
0.3316 \\
0.7194 \\
0.2369\end{array}$ & $\begin{array}{ll}0.0650 & \mathrm{Pt} \\
0.0272 & \mathrm{Pt} \\
0.2650 & \mathrm{MO} \\
0.5749 & \mathrm{MO}_{3} \\
0.1895 & \mathrm{MO}_{3}\end{array}$ & $\mid \begin{array}{c}3.92\left(\mathrm{NH}_{4}\right)_{2} \mathrm{O} \\
3.63\left(\mathrm{NH}_{4}\right)_{2} \mathrm{O} \\
79.92 \mathrm{MO}_{3} \\
79.91 \mathrm{MO}_{3} \\
79.98 \mathrm{MO}_{3}\end{array}$ & $\left\{\begin{array}{l}3.77 \%\left(\mathrm{NH}_{4}\right)_{2} \mathrm{O} \\
79.94 \% \mathrm{MO}_{3}\end{array}\right.$ & $3.62 \%\left(\mathrm{NH}_{4}\right)_{3} \mathrm{O}$ \\
\hline & & Differenz: & $16.29 \% \mathrm{H}_{2} \mathrm{O}$ & $16.27 \% \mathrm{H}_{2} \mathrm{O}$ \\
\hline
\end{tabular}

1 Analyse von D. Asch. 
Nach diesen Versuchen sind die in der 1. Mitteilung für das schwefligmolybdänsaure Kali und Natron aufgestellten Formeln zu streichen. Die Zusammensetzung der Alkali- und Erdalkalisalze wird durch die allgemeine Formel

$$
2 \mathrm{R}_{2} 0.2 \mathrm{SO}_{2} .5 \mathrm{MO}_{3}+\mathrm{xH}_{2} \mathrm{O}
$$

ausgedrückt. Nur das Ammoniumsalz tritt noch in einer anderen stabileren Form von der Zusammensetzung

$$
3\left(\mathrm{NH}_{4}\right)_{2} \mathrm{O}_{2} .2 \mathrm{SO}_{3} .8 \mathrm{MO}_{3}+5 \mathrm{H}_{2} \mathrm{O}
$$

auf. Für dies abweichende Verhalten des Ammoniumsalzes finden sich bei den komplexen anorganischen Säuren mehrfache Analogien. Von gewisser Wichtigkeit ist diese vorliegende Beobachtung aber vor allem für eine bisher noch nicht erledigte Frage hinsichtlich der Zusammensetzung der paramolybdänsauren Salze.

Für diese Verbindungen wird im allgemeinen die Formel

$$
3 \mathrm{R}_{2} \mathbf{0 . 7 \mathrm { MO } _ { 8 }}+\mathbf{x} \mathrm{H}_{2} \mathbf{O}
$$

angenommen. Von einer Reihe von Forschern ist eine andere, in den prozentischen Werten nahezu mit dieser übereinstimmende Zusammensetzung für die Körper vertreten worden, die ihren Ausdruck in der Formel

$$
5 \mathrm{R}_{2} 0.12 \mathrm{MO}_{3}+\mathrm{xH}_{2} \mathrm{O}
$$

findet. Besonders für das Ammoniumparamolybdat, das sich in mancher Beziehung von den übrigen Paramolybdaten unterscheidet, ist in den letzten Jahren die zweite Formel mehr eingeführt worden.

C. FrIEDHeim hat gemeinsam mit einigen Schülern ${ }^{1}$ die Frage aufzuklären gesucht, welche Zusammensetzung dem chemischen Verhalten der Paramolybdate am besten entspricht, indem er die Umsetzungsprodukte der Alkalimolybdate mit Erdalkali- und einigen Metallchloriden untersuchte, ohne zu einem definitiven Ergebnisse $\mathrm{zu}$ gelangen.

Die hier gemachten Beobachtungen scheinen nun dafür zu sprechen, dafs dem Ammoniumparamolybdat thatsächlich eine andere Zusammensetzung zukommt als den anderen Alkaliparamolybdaten; denn während aus den anderen Alkalimolybdaten durch schweflige Säure die Körper $2 \mathrm{R}_{2} \mathrm{O} .2 \mathrm{SO}_{2} .5 \mathrm{MO}_{3}+\mathrm{XH}_{2} \mathrm{O}$ erhalten wurden, ergab

1 F. Westphal, Inaug.-Dissertation, Berlin 1895. E. Marcrwald, Inaug.Dissertation, Basel 1895. 
Ammoniumparamolybdat das $\mathrm{Salz} 3 \mathrm{R}_{2} \mathrm{O} .2 \mathrm{SO}_{2}, 8 \mathrm{MO}_{3}+5 \mathrm{H}_{2} \mathrm{O}$. Allerdings konnte durch Umsetzung mit Chlorammonium das den übrigen Alkalisalzen entsprechende Ammoniumsalz erhalten werden; doch war dasselbe aufserordentlich unbeständig.

Diese beiden Reihen der schwefligmolybdänsauren Salze stehen nun in sehr ähnlichen Beziehungen zu den beiden für die Paramolybdate aufgestellten Formeln, wie aus den folgenden beiden Gleichungen hervorgeht.
1) $2 \mathrm{R}_{2} \mathrm{O} .2 \mathrm{SO}_{2} .5 \mathrm{MO}_{3}=3 \mathrm{R}_{2} \mathrm{O} .7 \mathrm{MO}_{3}+2 \mathrm{SO}_{2}-\mathrm{R}_{2} \mathrm{O} .2 \mathrm{MO}_{3}$.
2) $3 \mathrm{R}_{2} \mathrm{O} .2 \mathrm{SO}_{2} \cdot 8 \mathrm{MO}_{3}=5 \mathrm{R}_{2} \mathrm{O} .12 \mathrm{MO}_{3}+2 \mathrm{SO}_{2}-2\left(\mathrm{R}_{2} \mathrm{O} .2 \mathrm{MO}_{3}\right)$.

Der ersten Gleichung entsprechen die aus dem Kali- und Natronparamolybdat erhaltenen Produkte, der zweiten das stabile Ammoniumsalz, so dals hiernach dem Ammoniumparamolybdat wahrscheinlich die Zusammensetzung $5\left(\mathrm{NH}_{4}\right)_{2} \mathrm{O} .12 \mathrm{MO}_{3}+7 \mathrm{H}_{2} \mathrm{O}$ zuzuschreiben ist, während die übrigen Alkalimolybdate nach der Formel $3 R_{2} O$. $7 \mathrm{MO}_{3}+\mathrm{xH}_{2} \mathrm{O}$ zusammengesetzt sind.

Friedneim hat in den oben angeführten Arbeiten im Anschlusse an eine frühere Publikation ${ }^{1}$ über die entsprechenden Wolframate Konstitutionsformeln für diese verschiedenen Molybdate aufgestellt. Den beiden Zusammensetzungen der Paramolybdate entsprechen die Formeln

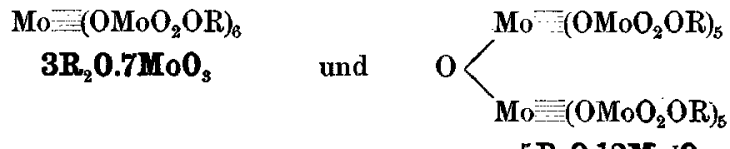

$$
\begin{aligned}
& 5 \mathrm{R}_{2} 0.12 \mathrm{Mo}^{1} \mathrm{O}_{3} \text {. }
\end{aligned}
$$

Aus diesen Strukturformeln liel'sen sich in einfacher Weise die schwefligmolybdänsauren Salze durch Ersetzung einiger Molybdänsäureketten durch Sulfonsäurereste ableiten; doch soll hierauf nur mit aller Reserve hingewiesen werden, da derartige Formeln erst der physikalisch-chemischen Begründung bedürfen, die bei der leichten Zersetzbarkeit der Molybdänsulfite in Lösung vorläufig experimentell noch nicht ausführbar war.

Dafs in diesen Verbindungen komplexe Körper vorliegen, kann allerdings auch ohne Leitfähigkeits- und Überführungsbestimmungen aus zahlreichen Reaktionen gefolgert werden, so vor allem aus der

1 Landenburg's Handwörterbuch (Artikel Wolfram), S. 245. 
leichten Löslichkeit der Erdalkalisalze, die bei nicht komplexen Molybdaten meist unlösliche Niederschläge darstellen.

Folgerungen auf die Struktur der schwefligen Säure aus diesen Verbindungen zu ziehen, wird eine spätere Veröffentlichung, die in der Ausführung begriffen ist, Gelegenheit bieten.

Herrn J. KoppeL, der ihn bei der Ausführung dieser Versuche unterstützt hat, sagt der Verf. für die ausgezeichnete Hilfe seinen besten Dank.

Wissenschaftl. chem. Laboratorium Berlin N., 12. Juni 1897.

Bei der Redaktion eingegangen am 14. Juni 1897. 